\title{
An organic charge trapping memory transistor with bottom source and drain contacts
}

\author{
Maarten Debucquoy, ${ }^{1, a), b)}$ Dieter Bode, ${ }^{1, b)}$ Jan Genoe, ${ }^{1}$ Gerwin H. Gelinck, ${ }^{2}$ and \\ Paul Heremans ${ }^{1, b)}$ \\ ${ }^{1}$ IMEC, Kapeldreef 75, B-3001 Leuven, Belgium \\ ${ }^{2}$ TNO-Holst Centre, High Tech Campus 31, 5656 AE Eindhoven, The Netherlands
}

(Received 28 July 2009; accepted 13 August 2009; published online 10 September 2009)

\begin{abstract}
We present an organic charge trapping memory transistor with lithographically defined bottom source and drain contacts. This device can be written and erased at voltages as low as $15 \mathrm{~V}$. More than 500 write and erase cycles and the retention of the trapped charge over more than three months are shown, demonstrating the possibilities of this device as a reprogramable nonvolatile organic memory element. (c) 2009 American Institute of Physics. [doi:10.1063/1.3223588]
\end{abstract}

Organic circuits are growing in complexity. Several hundred organic transistors are combined with high frequency diodes in transponder circuits for radio frequency identification (RFID) tags, several thousand are integrated with photodiodes in optical sensor arrays, and more then 10000 are used to drive active-matrix organic light-emitting diode displays. ${ }^{1-4}$ It is expected that these and other examples will lead to electronic applications on large and flexible substrates as well as low-cost electronics. A missing electronic building block is a reliable, nonvolatile, and electrically reprogramable memory device that can be integrated ("embedded") with the transistors of digital logic. This would open up applications such as organic reprogramable RFID tags or sensors capable of storing the read information. A possible candidate for such a memory element is an organic charge trapping memory transistor. This device is technologically compatible in terms of materials and device structure with logic-type organic transistors. Unfortunately, the charge trapping transistors reported so far have top source and drain electrodes patterned by shadow masks. Therefore, they cannot be integrated in circuits, neither be downscaled.

In early examples of organic charge trapping memory transistors, charges were trapped in an organic gate dielectric. $^{5-7}$ Relatively high programing voltages and long programing times were needed. Later structures used a dielectric stack of an oxide and an organic dielectric, ${ }^{8,9}$ with short programing pulses $\sim 1 \mu$ s but still with relatively large programing voltages $>50 \mathrm{~V}$. In more complex structures the trapping centers were metal nanoparticles at or near to the gate dielectric-semiconductor interface, ${ }^{10-12}$ organic molecules dissolved in a polymer matrix ${ }^{13}$ or a stack of oxides. ${ }^{14}$ Some of these show low programing voltages, but not in combination with sufficiently long data retention. Only recently, we demonstrated that low programing voltages, short programing pulses, and a good retention can be achieved in charge trapping memory transistors with a simple dielectric stack of an oxide and an organic dielectric, still with top source and drain contacts. ${ }^{15}$

Building on those results, we realized now an organic charge trapping memory transistor with a stacked dielectric

\footnotetext{
a)Electronic mail: maarten.debucquoy@imec.be.

${ }^{b)}$ Also at the Electrical Engineering Department, Katholieke Universiteit Leuven, Leuven, Belgium.
}

structure and lithographically defined bottom source and drain contacts. We show that these devices can be written and erased with short pulses at low programing voltages and that data is retained over more than 3 months. Moreover, several hundred write and erase cycles are demonstrated. This demonstrates the possibilities of this device as a reprogramable and embedded nonvolatile memory element.

The transistors in this study have a bottom gate electrode and bottom source and drain electrodes, as depicted in Fig. 1. The substrate is a highly $n$-doped $\mathrm{Si}$ wafer with $20 \mathrm{~nm}$ of thermally grown $\mathrm{SiO}_{2}$ on top. The highly $n$-doped $\mathrm{Si}$ serves as the gate electrode. Gold source and drain electrodes are patterned on top of the $\mathrm{SiO}_{2}$ by a lithographic process. Next, these samples are dipped in a $3,3,4,4,5,5,6,6$, $7,7,8,8,9,9,10,10,10$-heptadecafluoro-1-decanethiol solution in ethanol to form a monolayer of this thiol on top of the $\mathrm{Au}$ electrodes (Fig. 1). After rinsing the samples with ethanol, a $4 \mathrm{~nm}$ thick layer of poly(alpha-methylstyrene) ( $\mathrm{P} \alpha \mathrm{MS})$ is deposited by spin coating a 0.1 wt $\% \mathrm{P} \alpha \mathrm{MS}$ solution in toluene at $4000 \mathrm{rpm}$. In this process, the $\mathrm{P} \alpha \mathrm{MS}$ is repelled from the thiolated metal contacts, and is confined spontaneously to the channel region of the transistor. ${ }^{16}$ The stack of $\mathrm{SiO}_{2}$ and $\mathrm{P} \alpha \mathrm{MS}$ forms the gate dielectric. As a last step, a $40 \mathrm{~nm}$ thick layer of pentacene is deposited by organic molecular beam deposition ( $p \sim 10^{-8}$ Torr). The deposition rate and the substrate temperature during pentacene deposition are $0.25 \AA / s$ and $68{ }^{\circ} \mathrm{C}$, respectively. The transistors have channel lengths $L=10 \mu \mathrm{m}$ and channel width $W=1000 \mu \mathrm{m}$. The initial hole mobility extracted from the linear regime, $\mu_{h, \text { init }}$ and the ini-

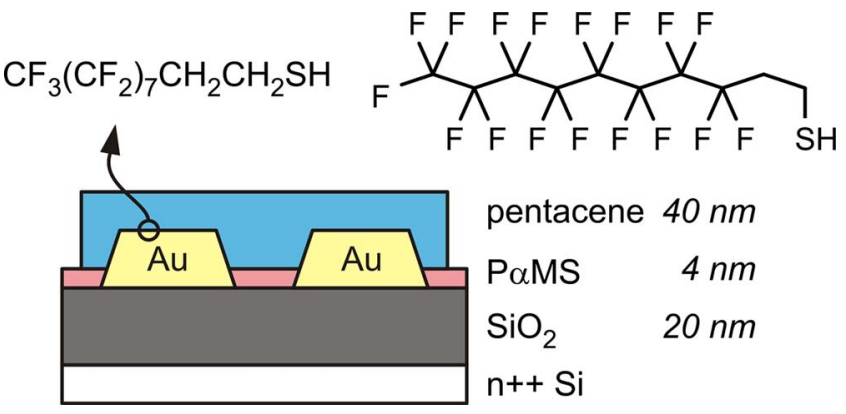

FIG. 1. (Color online) Schematic view of a bottom contact memory transistor, together with the chemical structure of the heptadecafluoro-1decanethiol, employed on the source and drain electrodes. 


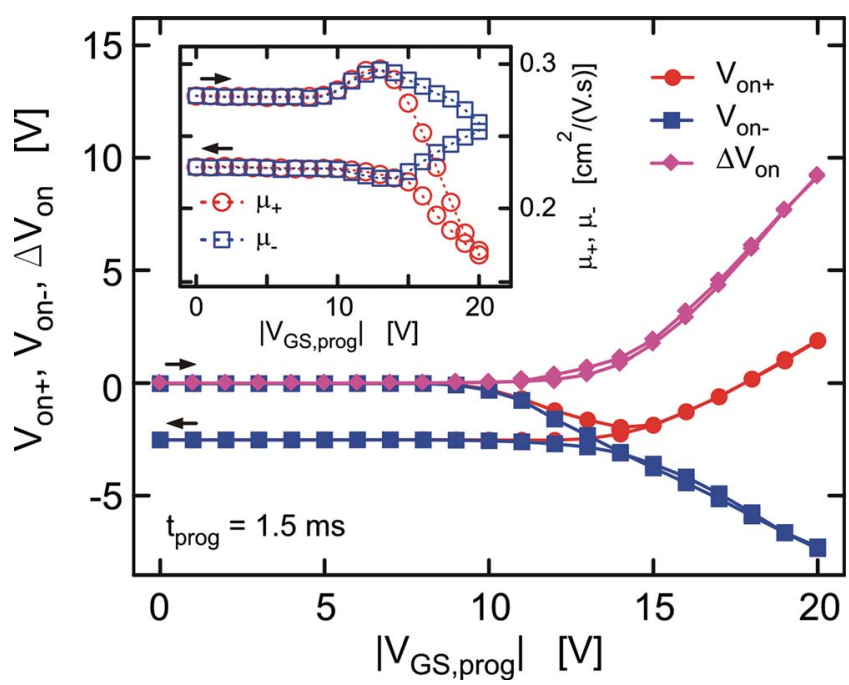

FIG. 2. (Color online) $V_{\text {on- }}, V_{\text {on }}$, and $\Delta V_{\text {on }}$ as a function of $\left|V_{\text {GS,prog }}\right|$ for $t_{\text {prog }}=1.5 \mathrm{~ms}$. The inset shows the hole mobility in the two memory states, as a function of $\left|V_{\mathrm{GS}, \text { prog }}\right|$ for $t_{\mathrm{prog}}=1.5 \mathrm{~ms}$.

tial threshold voltage $V_{T \text {.init }}$ are typically $\sim 0.3 \mathrm{~cm}^{2} /(\mathrm{V} \mathrm{s})$ and $\sim-0.3 \mathrm{~V}$.

Electrical data are obtained using two computer controlled Keithley 2602 units in a darkened nitrogen glovebox. Samples were not subjected to air. The measurement protocol is described elsewhere but briefly repeated here. ${ }^{15}$ First, the initial characteristics were measured to determine the initial turn-on voltage, $V_{\text {on,init }}$, of the device. The turn-on voltage $V_{\text {on }}$ is defined as the gate-source voltage $V_{\mathrm{GS}}$ at which the drain current $I_{\mathrm{D}}$ reaches $100 \mathrm{pA}$. Then, the device is subjected to a write pulse with pulse length $t_{\text {prog }}$ and a negative programing voltage $-V_{\mathrm{GS}, \text { prog. }}$. The erase pulse has the same length $t_{\text {prog }}$, as the write pulse, and a positive programing voltage $V_{\mathrm{GS}, \text { prog }}$, equal but opposite to the programing voltage of the write pulse. The drain-source voltage during programing, $V_{\mathrm{DS} \text {,prog }}$, is always kept at $0 \mathrm{~V}$. After a pulse, a small part of the transfer characteristics is measured to determine $V_{\text {on }}$. We denote by $V_{\text {on- }}$ the turn-on voltage after a negative write pulse, and $V_{\text {ont }}$ is the turn-on voltage after a positive erase pulse. $\Delta V_{\text {on }}$ is defined as the difference between $V_{\text {on+ }}$ and $V_{\text {on- }}$. These measurement steps can then be repeated for different $\left|V_{\mathrm{GS}, \text { prog }}\right|$.

In Fig. 2, $V_{\text {on- }}, V_{\text {on+ }}$, and $\Delta V_{\text {on }}$ are plotted as a function of $\left|V_{\mathrm{GS} \text {,prog }}\right|$, with $\left|V_{\mathrm{GS} \text {,prog }}\right|$ increasing from 0 to $20 \mathrm{~V}$ in steps of $1 \mathrm{~V}$. The observed shift in turn-on voltage at high programing voltages can be explained by trapping of holes and electrons in the gate dielectric stack. ${ }^{8,15}$ At low programing voltages $\left(\left|V_{\mathrm{GS}, \text { prog }}\right|<10 \mathrm{~V}\right), V_{\mathrm{on}-}=V_{\mathrm{on}+}=V_{\text {on,init }}$, and $\Delta V_{\text {on }}$ $=0 \mathrm{~V}$. At these voltages, the electric field over the $\mathrm{P} \alpha \mathrm{MS}$ is too low for the holes and electrons to be injected into the dielectric stack and as a result no charges are trapped. For values of $\left|V_{\mathrm{GS} \text {,prog }}\right|$ between 10 and $14 \mathrm{~V}, V_{\text {on- }} \approx V_{\text {on+ }}$ $<V_{\text {on,init }}$. Holes can tunnel into the dielectric stack during the negative write pulse, resulting in a more negative $V_{\text {on- }}$. But these trapped holes cannot be removed, nor be overwritten by electrons during the positive erase pulse. ${ }^{15}$ From $14 \mathrm{~V}$ on, the transistor can be programmed between two distinct states with different $V_{\text {on }}$. During a negative write pulse, holes can be trapped in the gate dielectric leading to a more negative $V_{\text {on-. }}$. A positive erase pulse, on the other hand, leads to the trapping of electrons, overwriting the trapped holes and re- sulting in a more positive $V_{\mathrm{on}+}$. The higher $\left|V_{\mathrm{GS}, \text { prog }}\right|$, the more charges can be trapped and the higher $\Delta V_{\text {on }}$. After $\left|V_{\mathrm{GS}, \text { prog }}\right|$ reaches $20 \mathrm{~V}$, we repeat the measurement procedure but with $\left|V_{\mathrm{GS} \text {,prog }}\right|$ decreasing from 20 to $0 \mathrm{~V}$. The measured $V_{\text {on }+}, V_{\text {on- }}$, and $\Delta V_{\text {on }}$ lay on top of the first scan for $\left|V_{\mathrm{GS}, \text { prog }}\right|>14 \mathrm{~V}$, illustrating the reproducibility of the write and erase mechanism. Only at the end of the measurement $\left(\left|V_{\mathrm{GS}, \text { prog }}\right|<14 \mathrm{~V}\right), \quad V_{\text {on- }}$, and $V_{\text {on+ }}$ end up at a voltage slightly more negative than $V_{\text {on,init }}$ indicating that some net positive charge remains trapped.

Interestingly, the trapping of both holes and electrons also affects the transistor's hole mobility. We show in the inset in Fig. 2 the hole mobility extracted in the linear regime of the transistor, after the negative write $\left(\mu_{-}\right)$and positive erase pulses $\left(\mu_{+}\right)$. Two trends in $\mu_{+}$and $\mu_{-}$can be noticed when $\left|V_{\mathrm{GS} \text {,prog }}\right|$ is increased from 0 to $20 \mathrm{~V}$ and decreased back to $0 \mathrm{~V}$. First, when $\left|V_{\mathrm{GS}, \text { prog }}\right|$ increases above $14 \mathrm{~V}, \mu_{+}$ becomes smaller than $\mu_{-}$. This can be related to the trapping of electrons in the dielectric. Calhoun et al. ${ }^{17}$ reported that electrons trapped in the dielectric reduce the hole mobility in the channel, while holes trapped in the dielectric do not. This is again an indication that from $14 \mathrm{~V}$ on, electrons and holes are indeed alternately trapped in the dielectric stack. Second, there is an overall decrease in the hole mobility as a result of the repeated pulses at high $\left|V_{\mathrm{GS}, p r o g}\right|\left[\mu_{+}\right.$and $\mu_{-}$are reduced from 0.28 to $\left.0.23 \mathrm{~cm}^{2} /(\mathrm{V} \mathrm{s})\right]$. It is not clear if this overall decrease is mainly due to the trapping of either holes or electrons and the accompanying damage to the pentacene $/ \mathrm{P} \alpha \mathrm{MS}$ interface, but it can be reduced by limiting $\left|V_{\mathrm{GS}, \mathrm{prog}}\right|$.

To open the memory window at low $\left|V_{\mathrm{GS}, p r o g}\right|$, the trapping of both holes and electrons is needed. ${ }^{13}$ At $\left|V_{\mathrm{GS}, \text { prog }}\right|$ $=15 \mathrm{~V}, \Delta V_{\text {on }}$ is almost $2 \mathrm{~V}$ corresponding to a ratio of the drain currents in the two memory states, $I_{\mathrm{D}+} / I_{\mathrm{D}}$, of more than $200\left(I_{\mathrm{D}+}\right.$ and $I_{\mathrm{D}-}$ measured at $V_{\mathrm{GS}, \text { sense }}=-1.5 \mathrm{~V}$ and $\left.V_{\mathrm{DS} \text {,sense }}=-0.02 \mathrm{~V}\right)$.

Figure 3(a) shows the evolution of $V_{\mathrm{on}-}, V_{\mathrm{on}+}$, and $\Delta V_{\text {on }}$ with the increasing number of cycles (one cycle being a combination of a write and an erase pulse). After more than 500 cycles at $\left|V_{\mathrm{GS}, \text { prog }}\right|=15 \mathrm{~V}$ and $t_{\text {prog }}=1.5 \mathrm{~ms}, \Delta V_{\text {on }}$ decreases from 1.9 to $1.4 \mathrm{~V}$. As important is the evolution of $I_{\mathrm{D}+} / I_{\mathrm{D}-}$, depicted in Fig. 3(b) $\left(I_{\mathrm{D}+}\right.$ was measured at $V_{\mathrm{GS} \text {,sense }}=-1.5 \mathrm{~V}$ and $V_{\mathrm{DS} \text {,sense }}=-0.02 \mathrm{~V}$. $I_{\mathrm{D}-}$ was taken fixed at $10 \mathrm{pA}$ to exclude variations in $I_{\mathrm{D}+} / I_{\mathrm{D}-}$ due to the noise in the cutoff current of the transistor). As the inset of Fig. 3(b) shows, the variations in $I_{\mathrm{D}+} / I_{\mathrm{D}-}$ are caused by a slight shift in $V_{\text {ont }}$ and $V_{\text {on- }}\left(I_{\mathrm{D}+} / I_{\mathrm{D}-}\right.$ rises $)$ and an overall decrease in the hole mobility $\left(I_{\mathrm{D}+} / I_{\mathrm{D}-}\right.$ decreases $)$. After more than 500 cycles, $I_{\mathrm{D}+} / I_{\mathrm{D}-}>130$, and the device still works as a memory transistor with two distinct states.

The retention of the trapped charges is another important figure of merit, shown in Fig. 4. After a write pulse with $-V_{\mathrm{GS}, \text { prog }}=-20 \mathrm{~V}$ and $t_{\text {prog }}=1.5 \mathrm{~ms}, V_{\text {on }}$ was measured at different time intervals. The same was done on another device on the same sample after an erase pulse with $V_{\mathrm{GS}, \text { prog }}$ $=20 \mathrm{~V}$ and $t_{\text {prog }}=1.5 \mathrm{~ms}$. In between the measurements, the sample was left in a darkened nitrogen glovebox, without applying any voltages to the electrodes. Each data point is the average of measurements on at least two different devices. At this $\left|V_{\mathrm{GS}, \text { prog }}\right|$, the retention of both the trapped holes and the trapped electrons can be tracked. After 3 months, all trapped electrons are lost and $V_{\text {ont }}$ is reduced to 0 


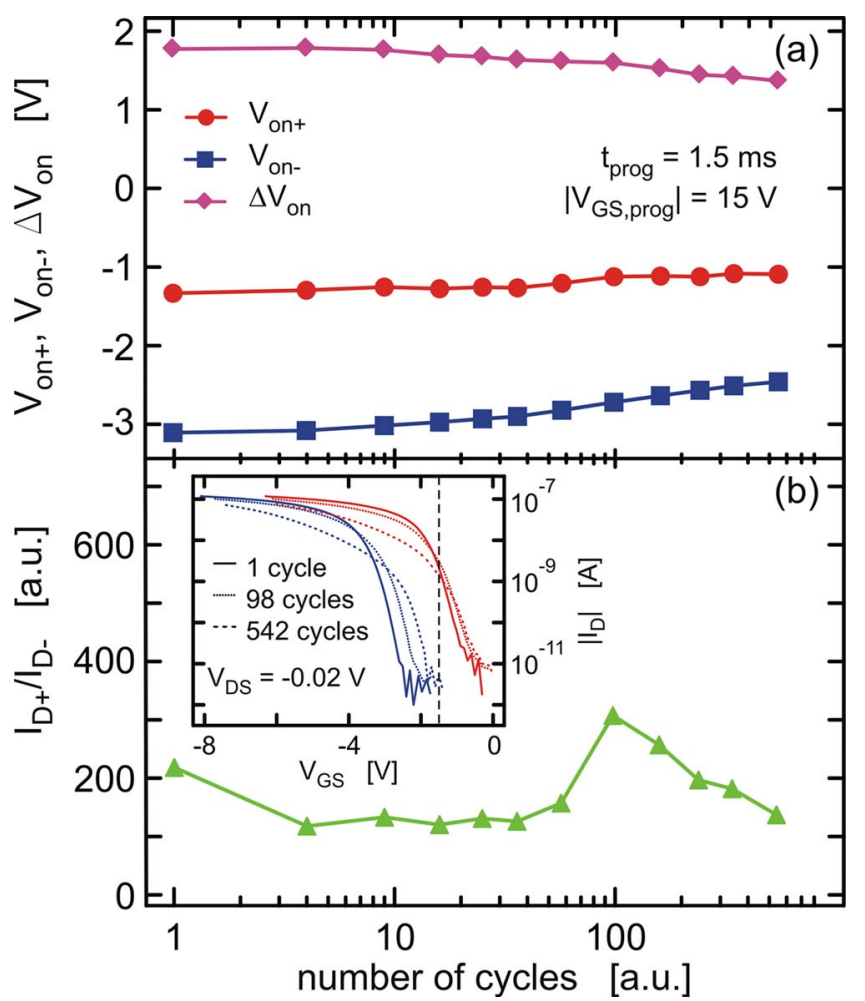

FIG. 3. (Color online) (a) $V_{\text {on- }}, V_{\text {on }}$, and $\Delta V_{\text {on }}$ as a function of the number of cycles for $t_{\text {prog }}=1.5 \mathrm{~ms}$ and $\left|V_{\mathrm{GS}, \text { prog }}\right|=15 \mathrm{~V}$. (b) $I_{\mathrm{D}+} / I_{\mathrm{D}-}$ as a function of the number of cycles for $t_{\text {prog }}=1.5 \mathrm{~ms}$ and $\left|V_{\mathrm{GS}, \text { prog }}\right|=15 \mathrm{~V}$. $I_{\mathrm{D}}$ was measured at $V_{\mathrm{GS} \text {,sense }}=-1.5 \mathrm{~V}$ and $V_{\mathrm{DS} \text {,sense }}=-0.02 \mathrm{~V}$. The inset shows the transfer characteristics in the two memory states after different numbers of cycles.

V. $V_{\text {on- }}$ on the other hand, tends to saturate toward $-2 \mathrm{~V}$, indicating that the holes are trapped in a trap distribution that contains also deep traps. These deeply trapped holes result in a good retention but, as we showed in Fig. 2, can be over-

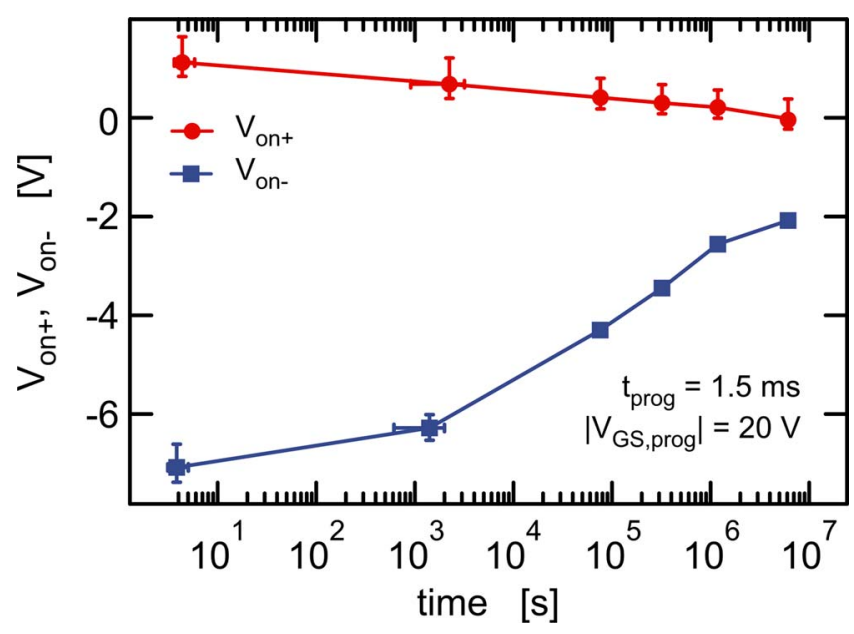

FIG. 4. (Color online) Retention measurement for a bottom contact memory transistor. The first point of the $V_{\text {on- }}$ curve is reached after a write pulse with $-V_{\mathrm{GS}, \text { prog }}=-20 \mathrm{~V}$ and $t_{\mathrm{prog}}=1.5 \mathrm{~ms}$. The first point of the $V_{\mathrm{on}+}$ curve is reached after an erase pulse with $V_{\mathrm{GS}, \text { prog }}=20 \mathrm{~V}$ and $t_{\text {prog }}=1.5 \mathrm{~ms}$. written by electrons during an erase pulse with $V_{\mathrm{GS}, \text { prog }}$ $>14 \mathrm{~V}$. The good retention of the trapped holes confirms the possibility to use this device as a nonvolatile memory element.

In summary, we realized an organic charge trapping memory transistor with bottom source and drain contacts. Holes can be trapped in the gate dielectric during a write pulse of $1.5 \mathrm{~ms}$ at $-15 \mathrm{~V}$. These trapped holes can be erased by overwriting them by electrons with a pulse of $1.5 \mathrm{~ms}$ at $15 \mathrm{~V}$. More than 500 cycles have been measured with an $I_{\mathrm{D}+} / I_{\mathrm{D}-}$ systematically larger than 100 and the retention of trapped holes was shown to be longer than 3 months. The compatibility of the device fabrication and structure with that of logic-type transistors and its promising figures of merit indicate that this device type is an interesting candidate for nonvolatile memory embedded with plastic logic.

The authors would like to thank Peter Vicca and Steve Smout for their support during the processing in general and the lithographic steps in particular. M.D. thanks the Institute for the Promotion of Innovation through Science and Technology in Flanders (IWT-Vlaanderen) for financial support.

${ }^{1}$ K. Myny, M. Beenhakkers, N. van Aerle, G. H. Gelinck, J. Genoe, W. Dehaene, and P. Heremans, Dig. Tech. Pap.-IEEE Int. Solid-State Circuits Conf. 2009, 206 (2009).

${ }^{2}$ G. H. Gelinck, H. E. A. Huitema, E. Van Veenendaal, E. Cantatore, L. Schrijnemakers, J. B. P. H. Van der Putten, T. C. T. Geuns, M. Beenhakkers, J. B. Giesbers, B. H. Huisman, E. J. Meijer, E. M. Benito, F. J. Touwslager, A. W. Marsman, B. J. E. Van Rens, and D. M. De Leeuw, Nature Mater. 3, 106 (2004).

${ }^{3}$ I. Yagi, N. Hirai, M. Noda, A. Imaoka, Y. Miyamoto, N. Yoneya, K. Nomoto, J. Kasahara, A. Yumoto, and T. Urabe, SID Int. Symp. Digest Tech. Papers 200763.2 (2007).

${ }^{4}$ T. Someya, Y. Kato, S. Iba, Y. Noguchi, T. Sekitani, H. Kawaguchi, and T. Sakurai, IEEE Trans. Electron Devices 52, 2502 (2005).

${ }^{5}$ M. Mushrush, A. Facchetti, M. Lefenfed, H. E. Katz, and T. J. Marks, J. Am. Chem. Soc. 125, 9414 (2003).

${ }^{6}$ H. E. Katz, X. M. Hong, A. Dodabalapur, and R. Sarpeshkar, J. Appl. Phys. 91, 1572 (2002).

${ }^{7}$ T. B. Singh, N. Marjanovic, G. J. Matt, N. S. Sariciftci, R. Schwödlauer, and S. Bauer, Appl. Phys. Lett. 85, 5409 (2004).

${ }^{8}$ K. J. Baeg, Y. Y. Noh, J. Ghim, S. J. Kang, H. Lee, and D. Y. Kim, Adv. Mater. 18, 3179 (2006)

${ }^{9}$ K. J. Baeg, Y. Y. Noh, J. Ghim, B. Lim, and D. Y. Kim, Adv. Funct. Mater. 18, 3678 (2008).

${ }^{10}$ J. H. Kim, K. H. Baek, C. K. Kim, Y. B. Kim, and C. S. Yoon, Appl. Phys. Lett. 90, 123118 (2007).

${ }^{11}$ C. Novembre, D. Guérin, K. Lmimouni, C. Gamrat, and D. Vuillaume, Appl. Phys. Lett. 92, 103314 (2008).

${ }^{12}$ M. F. Mabrook, Y. Yun, C. Pearson, D. A. Zeze, and M. C. Petty, Appl. Phys. Lett. 94, 173302 (2009).

${ }^{13}$ W. Wu, H. Zhang, Y. Wang, S. Ye, Y. Guo, C. Di, G. Yu, D. Zhu, and Y. Liu, Adv. Funct. Mater. 18, 2593 (2008).

${ }^{14}$ M.-F. Chang, P.-T. Lee, S. P. McAlister, and A. Chin, Appl. Phys. Lett. 93, 233302 (2008).

${ }^{15}$ M. Debucquoy, M. Rockelé, J. Genoe, G. H. Gelinck, and P. Heremans, Org. Electron. 10, 1252 (2009).

${ }^{16}$ K. Myny, S. De Vusser, S. Steudel, D. Janssen, R. Muller, S. De Jonge, S. Verlaak, J. Genoe, and P. Heremans, Appl. Phys. Lett. 88, 222103 (2006).

${ }^{17}$ M. F. Calhoun, C. Hsieh, and V. Podzorov, Phys. Rev. Lett. 98, 096402 (2007) 